Unfallchirurg 2021 $124: 601-609$

https://doi.org/10.1007/s00113-021-01044-0

Angenommen: 14. Juni 2021

Online publiziert: 12. Juli 2021

(c) Der/die Autor(en) 2021

Redaktion

J. Lotz, Göttingen

M.-A. Weber, Rostock

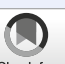

\title{
Diagnostik und Therapie traumatischer Aortenverletzungen
}

\author{
R. M. Benz ${ }^{1}$ V. Makaloski ${ }^{2} \cdot$ M. Brönnimann $\cdot$ N. Mertineit $\cdot$ H. von Tengg-Kobligk \\ ${ }^{1}$ Diagnostische, Interventionelle und Pädiatrische Radiologie, Inselspital, Universität Bern, Bern, Schweiz \\ ${ }^{2}$ Universitätsklinik für Herz- und Gefäßchirurgie, Inselspital, Universität Bern, Bern, Schweiz
}

\section{Zusammenfassung}

\section{In diesem Beitrag}
- Diagnostik Bildgebende Untersuchungen • Bildbe- funde
- Therapie Thoracic endovascular aortic repair vs. of- fene Versorgung • Thoracic endovascular aortic repair
- Resümee

Hintergrund: Traumatische Aortenverletzungen (TAV) sind seltene Folgen von stumpfen Traumata, die eine hohe Mortalität und Morbidität aufweisen. Die schnelle und akkurate Diagnostik sowie die Wahl der korrekten Therapie sind für das Patientenüberleben elementar.

Fragestellung: Bestimmung des aktuellen Standards der Abklärung von TAV im akuten Trauma-Setting und Evaluation der aktuellen Leitlinien zur Therapie.

Material und Methode: Eine Literaturrecherche wurde durchgeführt, mit der Suche nach Publikationen, die die Abklärung und Diagnostik der TAV beschreiben. Außerdem wurden Leitlinien für die Behandlung und Nachsorge von TAV zusammengefasst. Ergebnisse: In der Literatur wird trotz geringer Spezifität eine konventionelle Thoraxröntgenaufnahme als Initialdiagnostik genannt. Es sollte primär, als Modalität der Wahl, zur Diagnostik und zur Therapiestratifizierung eine Computertomographie (CT) aufgrund der hohen Sensitivität und Spezifität nachfolgen. In allen Leitlinien ist die thorakale endovaskuläre Aortenrekonstruktion ("thoracic endovascular aortic repair", TEVAR) die Therapie der Wahl bei höhergradigen TAV (Grade II-IV) und hat die offene Chirurgie in dem meisten Fällen abgelöst.

Schlussfolgerung: Nach einer kurzfristig erfolgten CT-Diagnostik und Einteilung wird die TEVAR der offenen Chirurgie bei therapiebedürftigen TAV vorgezogen.

\section{Schlüsselwörter}

Klassifikation - Computertomographische Angiographie · Thorakale endovaskuläre Aortenrekonstruktion · Offene Aortenchirurgie · Leitlinien

Zwar stellen traumatische Aortenverletzungen (TAV) seltene Ereignisse dar, aber aufgrund der hohen, mit ihnen assoziierten Morbidität und Mortalität ist die schnelle und akkurate Diagnostik für die Prognose des Patienten ausschlaggebend. Neben den traditionell-chirurgischen Therapieverfahren, die ebenfalls mit einer entsprechenden Morbidität und Mortalität einhergehen, haben sich in den letzten Jahrzenten zunehmend minimal-invasive Vorgehensweisen als Erstlinientherapie durchgesetzt und wurden in den nordamerikanischen und europäischen Guidelines verankert $[10,12,21,24,28,37,43,47]$.

\section{Hintergrund}

Die Mehrzahl der TAV ist durch stumpfe Traumata im Rahmen von Verkehrsunfällen, die in Form einer Kombination von Zug und Scherverletzung auftreten, verursacht [38]. Hierbei kann sowohl die thorakale als auch die abdominale Aorta verletzt werden, allerdings tritt Letzteres selten auf [7, 39]. Aufgrund der überwiegenden Mehrheit der thorakalen Aortenverletzungen bilden diese den Schwerpunkt des vorliegenden Beitrags. Sowohl in der Aorta abdominalis als auch in der Aorta thoracica kommen die Verletzungen an Prädilektionsstellen vor, was für die Unterscheidung einer akuten Traumafolge von einem chro- 
Tab. 1 Prädilektionsstellen traumatischer Aortenverletzungen [7,31, 40]

\begin{tabular}{l|l} 
Thorax & Abdomen \\
\hline $\begin{array}{l}\text { Aortenisthmus } \\
(90 \%)\end{array}$ & $\begin{array}{l}\text { A. mesenterica inferior } \\
(\sim 33 \%)\end{array}$ \\
\hline $\begin{array}{l}\text { Aorta ascen- } \\
\text { dens }(5 \%)\end{array}$ & Nierenarterien $(\sim 25 \%)$ \\
\hline $\begin{array}{l}\text { Hiatus des } \\
\text { Diaphragmas } \\
(5 \%)\end{array}$ & $\begin{array}{l}\text { Unterhalb der A. mesen- } \\
\text { terica inferior }(\sim 20 \%)\end{array}$ \\
\hline
\end{tabular}

nischen Geschehen, wie z. B. einer arteriopathischen Dissektion, relevant ist.

\section{》) Die schnelle und akkurate} Diagnostik stellt einen dringlichen Beitrag zur erfolgreichen Therapie dar

Ungefähr $80 \%$ der Patienten mit TAV erreichen das Krankenhaus nicht lebend. Die Mortalität beträgt aber auch bei Patienten mit technisch erfolgreicher Therapie noch bis zu $30 \%$ [19]. Diese Zahlen unterstreichen die Dringlichkeit einer schnellen und akkuraten Diagnostik. Abhängig vom Schweregrad und von der Gefäßanatomie können die TAV konservativ, chirurgisch oder endovaskulär behandelt werden [34, 38, 41, 47]. Die endovaskuläre Therapie ist auch im deutschsprachigen Raum als „thoracic endovascular aortic repair" (TEVAR) bekannt. Die Wahl des richtigen Therapieverfahrens, der Zeitpunkt der Therapie, aber auch eine begleitende Antikoagulation sind Gegenstand einer interdisziplinären Entscheidung und individuell auf dem Boden von Patientenalter, Komorbidität, Begleitverletzungen und Zustand des Patienten zu festzulegen [30, 41, 47].

\section{Diagnostik}

\section{Bildgebende Untersuchungen}

Obwohl eine konventionelle Thoraxröntgenuntersuchung in vielen Zentren zum initialen Screening gehört, ist diese aufgrund der niedrigen Spezifität von etwa $10 \%$ und der fehlenden direkten Darstellung der Gefäße zur Diagnostik und zur Einteilung der TAV nicht geeignet [7, 9, 12]. Befunde im konventionellen Röntgenbild sind eine Verbreiterung des Mediastinums, der paraspinalen und paratrachealen Zone, eine abnorme Aortenkontur, eine Kom-
Tab. 2 Einteilung der Aortenverletzungen in 4 Grade

Grad Aortenverletzung

I Intimariss

II Intramurales Hämatom

\begin{tabular}{|l|l|}
\hline III & Pseudoaneurysma \\
\hline IV & Ruptur \\
\hline
\end{tabular}

pression oder Verlagerung der Luftwege, ein Verlust des aortopulmonalen Fensters, ein großer linksseitiger Pleuraerguss oder eine Verbreiterung des linken supraapikalen Weichteilschattens [19].

Im Schockraum hat sich als Initialdiagnostik nach Traumata die bettseitig durchgeführte Extended Focused Assessment with Sonography for Trauma (EFAST) zunehmend durchgesetzt. Diese ist allerdings zur TAV-Beurteilung ebenfalls nicht geeignet [19].

\section{》) Mithilfe der CTA können essen- zielle und akut therapierelevante Verletzungen dargestellt werden}

In den meisten Zentren wird nach einer globalen Initialbeurteilung kurzfristig eine Kontrastmittel(KM)-gestützte computertomographische Angiographie (CTA) durchgeführt. Gründe sind die Verfügbarkeit, die Geschwindigkeit der Untersuchung sowie die Spezifität (fast 100\%) und hohe Sensitivität (96\%) [42]. Die detaillierten Bildakquisitionsprotokolle variieren zwischen den Häusern und sind von den jeweiligen Gegebenheiten abhängig. Sie beinhalten aber meist eine native $\mathrm{CT}$ und eine arterielle KM-Phase sowie fakultativ eine portalvenöse und/oder eine KM-Spätphase. In der Basis geht es darum, essenzielle und akut therapierelevante Verletzungen wie Frakturen, Organund Gefäßverletzung sowie arterielle oder venöse Blutungen schnell und akkurat zu diagnostizieren und möglichst exakt zu lokalisieren.

\section{Bildbefunde}

\section{Native Computertomographie} Indirekte Hinweise auf eine Aortenverletzung sind ein mediastinales Hämatom und/oder eine periaortale Fettgewebsim-
Tab. 3 Kriterien, die für eine endovasku läre oder eine chirurgische Versorgung der traumatischen Aortenverletzung sprechen Endovaskuläre Ver- $\quad$ Chirurgische sorgung Versorgung

\begin{tabular}{ll|l} 
Multiple schwere & Anatomische
\end{tabular}

Verletzungen

Keine Beteiligung der

Aorta ascendens

Schwere rechtseitige

Thorax- oder Lungenverletzung (Intoleranz einer Intubation

Geringe Lebenserwar-

tung

Multiple Komorbiditäten

$T E V A R$ "thoracic endovascular aortic repair"

bibierung, v.a. wenn sie an den Prädilektionsstellen für TAV auftreten (•Tab. 1; - Abb. 1, 2b, 3a und 4a,b). Diese Prädilektionsstellen finden sich dort, wo die Aorta fixiert ist $[7,42]$.

Auch andere Thoraxverletzungen können einen indirekten Hinweis auf eine TAV darstellen [31]. Abgesehen davon bietet bereits die native $\mathrm{CT}$ die Möglichkeit, ein akutes Wandhämatom von artheriosklerotisch-thrombotischen Wandauflagerungen zu unterscheiden [17]. Um diesen Effekt gut ausnutzen zu können, darf der Hämatokritwert des Patienten nicht zu niedrig sein (Cave: verdünntes Blut), und ein erfahrener Radiologe kennt auch weitere Unterscheidungskriterien auf Basis der KMgestützten angiographischen Bildserie.

\section{Computertomographische Angiographie}

In der CTA, die mit jodhaltigem KM durchgeführt wird, wird die Aortenverletzung direkt dargestellt. Die Bildbefunde dienen zur Gradeinteilung der Aortenverletzung (- Tab. 2), die Konsequenzen für die Art und den Zeitpunkt der Therapie hat.

\section{॥ Die EKG-Triggerung ist zur} Diagnostik von Läsionen der Aortenwurzel und Aorta ascendens essenziell

Ausdruck einer traumatischen Aortenverletzung sind die nachfolgend beschriebenen Befunde, wenn sie an den Prädilektionsstellen ( $\mathbf{0}$ Tab. 1) auftreten. 


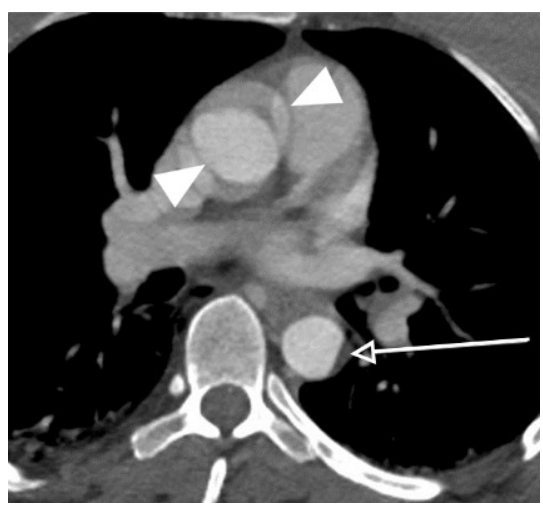

Abb. $1 \triangle$ Computertomographische Aufnahme einer 44-jährigen Patientin nach einem Verkehrsunfall mit sich überschlagendem Auto, CTPolytraumaspirale mit Split-Bolus-Kontrastmittelgabe. Akute, traumatische Aortenverletzung Grad I mit Intima-Flap (Pfeil) in der proximalen Aorta descendens. Arteriosklerotische Veränderungen sind als Differenzialdiagnose bei diesem fokalen, singulären Befund und dem niedrigen Patientenalter unwahrscheinlich. Die Patientin wurde konservativ therapiert

Ein intraluminaler Füllungsdefekt ist Ausdruck entweder eines Intimarisses (eher feinlinear; - Abb. 2b) oder eines Thrombus (eher breitlinear; - Abb. 1) oder von beidem. Eine abnorme Kontur der Aorta, im Sinne eines fokalen Kalibersprungs, ist entweder Zeichen eines muralen Hämatoms ( $\bullet$ Abb. 1 ) oder eines Pseudoaneurysmas (- Abb. 2a, 3a und 4b). Im schwerwiegendsten Fall ist ein KMAustritt erkennbar [7]. Die Differenzierung von Verkalkungen und $\mathrm{KM}$ ist mitunter schwierig, sodass eine vorangegangene native CT von Relevanz sein kann. Gegebenenfalls ist in der Spätphase eine Aussage über die Dynamik der Extravasation möglich, wobei die Therapie eines instabilen Patienten nicht aufgrund der bildgebenden Untersuchung verzögert werden sollte.

Einige nichtakute Bildbefunde können eine TAV vortäuschen. Dazu gehören ein Ductus diverticulum („ductus bump“), der sich v.a. durch einen breiten Hals gegen das eher schmalhalsige, traumatische Pseudoaneurysma abgrenzt. Im Fall eines Infundibulums, das sich als konische Ausstülpung darstellt, ist an dessen Spitze ein abgehendes Gefäß erkennbar. Auch Bewegungsartefakte können Dissektionen oder Konturunregelmäßigkeiten vortäuschen (- Abb. 1 und 3a). Die indirekten Zeichen können zur Differenzierung hilfreich sein

\section{Infobox 1}

Anatomische Kriterien für die Planung und Durchführung einer Thoracic endovascular aortic repair

- Aortendurchmesser $\geq 17,5 \mathrm{~mm}$ an der proximalen Landezone

- Proximale und distale Landezone $\geq 2 \mathrm{~cm}$ lang

- Geringe Kalzifikationen der Landezonen

- Fokale Läsion

- Keine signifikante Tortuosität der Aorta

- Zugangsgefäß mit adäquatem Durchmesser ohne relevante Stenosen oder Tortuosität

Radius des Aortenbogens

$[15,35]$. Daher ist eine EKG-Triggerung zur Diagnostik Läsionen im Bereich der Aortenwurzel und der Aorta ascendens essenziell [46].

Ein transösophageales Echokardiogramm stellt eine Alternative dar; diese erfolgt bei hämodynamisch instabilen, ventilierten Patienten, die eine unmittelbare Diagnostik benötigen. Der Vorteil besteht darin, dass es am Patientenbett angefertigt werden kann [16]. Allerdings kann ein Segment der Aorta ascendens aufgrund einer Überlagerung der Trachea und des rechten Hauptbronchus nicht eingesehen werden [12].

Die katheterbasierte i.a.-Angiographie zur Diagnostik von Aortenverletzungen wird in der heutigen Zeit kaum noch durchgeführt [12].

\section{Therapie}

Thoracic endovascular aortic repair vs. offene Versorgung

Sind die Initialdiagnostik und Bilanzierung der Verletzungen abgeschlossen, geht es darum, die Therapien und deren Reihenfolge anhand der Relevanz zu triagieren. Die invasive Versorgung ist bei einer TAV der Grade II-IV indiziert. Die Typ-I-Verletzung wird konservativ und mit engmaschiger Überwachung behandelt [34]. Im Fall eines Progresses können leichtgradige TAV im Verlauf ebenfalls interventionsbedürftig werden.

Die TEVAR ist in den meisten Fällen die Therapie der Wahl. Auch wenn die Notwendigkeit einer Langzeitüberwachung nach TEVAR von einigen Autoren kritisch beurteilt wird [22], werden die Patienten meist über einen langen Zeitraum nachverfolgt, mit entsprechender Strahlenbelastung, die sich v. a. bei jungen Patienten akkumuliert $[34,49]$. Ein Vorteil der offenen Versorgung ist das Wegfallen dieser Nachsorgeuntersuchungen. Allerdings birgt die chirurgische Therapie eine höhere Morbidität und Mortalität; dies wird im Abschn. Komplikationen detaillierter ausgeführt. Deswegen ist ein chirurgisches Vorgehen nur bei wenigen Patienten indiziert ( $\bullet$ Tab. 3) und wurde in den letzten Jahren zunehmend durch die in den meisten Guidelines empfohlene TEVAR ersetzt $[10,12,21,24,28$, $37,43,47]$.

》) Die überwiegende Zahl der Guidelines empfiehlt trotz Notwendigkeit der Langzeitüberwachung die TEVAR

Zur erfolgreichen Durchführung einer TEVAR sind gewisse anatomische Gegebenheiten Voraussetzung. Diese Kriterien müssen bei der Indikationsstellung und Planung der TEVAR berücksichtigt werden und sind in $\mathbf{D}$ Infobox 1 zusammengefasst [20].

Kotelis et al. haben basierend auf der bildgebenden Untersuchung verschiedene Aspekte einer Risikostratifizierung für die TEVAR vorgestellt, die im Einzelfall in Betracht gezogen werden können. Darunter fallen u.a. ein thrombogener und ein betont gotischer Aortenbogen [20, 25, 26].

\section{Thoracic endovascular aortic repair}

\section{Timing}

Wenn die Indikation zur TEVAR gestellt ist, wird sie abhängig vom Grad der Verletzung sofort oder im Verlauf durchgeführt [28, 34]. Den besten Therapiezeitpunkt zu definieren, ist allerdings meist Teil eines komplexen und interdisziplinären Entscheidungsprozesses, v.a., wenn die TAV mit anderen schweren Verletzungen, wie z.B. des Gehirns, des Beckens oder der parenchymatösen Oberbauchorgane, einhergeht. Bei instabilem Patienten wird die TEVAR dringlich durchgeführt. Im Fall des hämodynamisch stabilen Patienten streitet sich die Literatur allerdings über den genauen Zeitpunkt. So empfiehlt z.B. die Society for Vascular Surgery in ihren Guidelines eine möglichst schnelle 

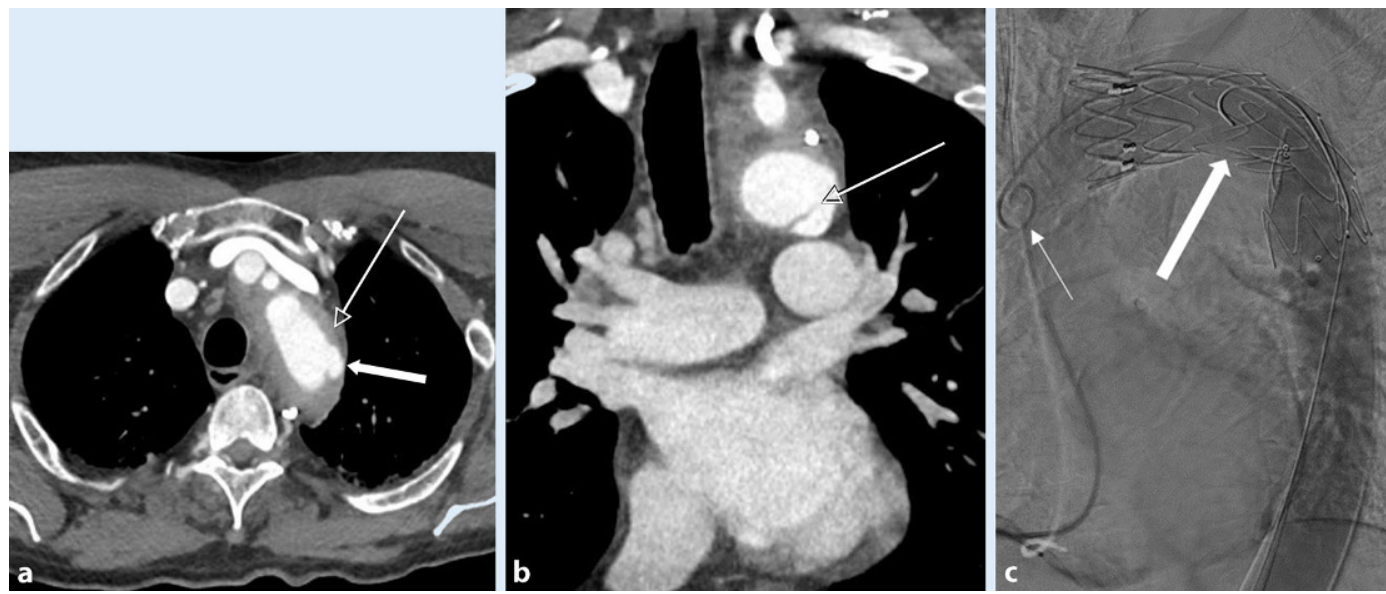

Abb. $2 \Delta$ Computertomographische Aufnahmen eines akuten, traumatischen Aortensyndroms Grad IV mit traumatischer Aortenruptur in loco classico und Typ-B-Dissektion, CT-Polytraumaspirale mit Split-Bolus-Kontrastmittelgabe. a Periaortales Hämatom (Pfeil) mit Aneurysma spurium (weißer Pfeil) des Aortenbogens. b Dissektionsmembran im Aortenbogen (Pfeil). c Versorgung mithilfe der "thoracic endovascular aortic repair" (TEVAR) mit unauffälliger intrainterventioneller, unter Durchleuchtung (DL) gesteuerter Kontrolle mit einem Pigtail-Katheter (Pfeil) und Stent-Körper (dickerPfeil)
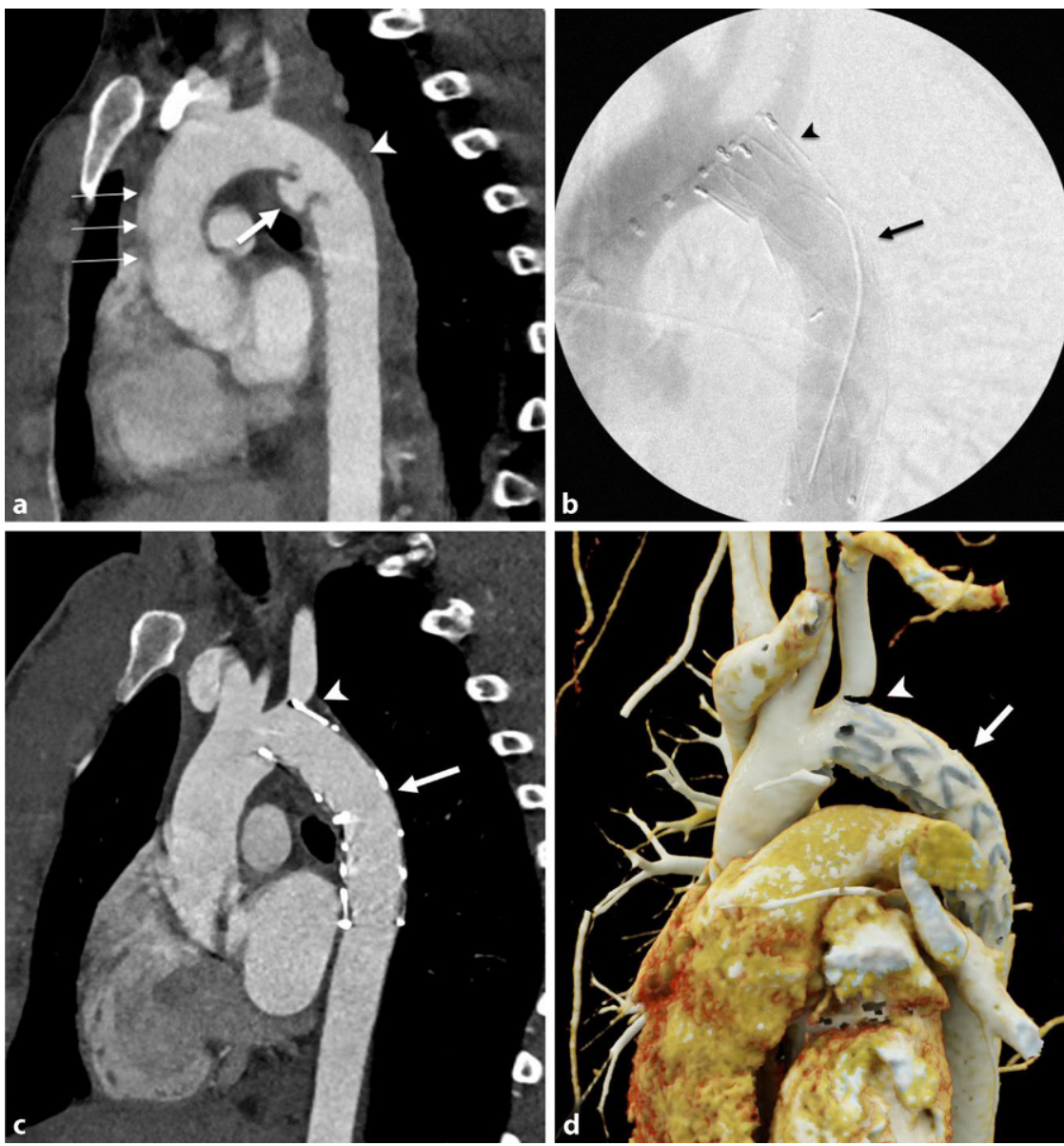

Abb. $3 \Delta$ Computertomographische Aufnahmen eines 28-jährigen Patienten mit Aortenruptur loco typico nach einem Hochgeschwindigkeitstrauma (Verkehrsunfall), CT-Polytraumaspirale mit SplitBolus-Kontrastmittelgabe. a Aortenruptur (Grad IV) ca. $1 \mathrm{~cm}$ distal des A.-subclavia-sinistra-Abgangs mit kleinem Intima-Flap (Pfeil) und Mediastinalhämatom (Pfeilspitze), b angiographische Kontrolle nach "thoracic endovascular aortic repair" (TEVAR; Pfeil); der "Uncovered"-Anteil des Stent-Grafts reicht knapp über den Abgang der A. subclavia sinistra (Pfeilspitze). c Die Verlaufskontrolle in der computertomographischen Angiographie (CTA) zeigt eine offene A. subclavia sinistra (Pfeilspitze) ohne Endoleak (Pfeil). d 3D-Rekonstruktion nach TEVAR (Pfeil) mit knapp überdeckter, aber regelrecht perfundierter A. subclavia sinistra (Pfeilspitze)
$(<24 \mathrm{~h})$ Durchführung der TEVAR [28]. Dagegen empfiehlt die Eastern Association for the Surgery of Trauma (EAST) eine TEVAR im Verlauf ( $>24 \mathrm{~h}$; [13]). Eine 2021 publizierte Arbeit, die die Daten von 2821 Patienten aus der amerikanischen Traumadatenbank analysierte, berichtete jedoch ein deutlich besseres Ergebnis bei angepasstem Schweregrad im Fall einer TEVAR nach $24 \mathrm{~h}$ [1]. Auch die Frage nach einer prä- und/oder interinterventionellen Heparinisierung ist Gegenstand von individuellen Erwägungen [30].

\section{Technik}

Die TEVAR wird unter Vollnarkose durchgeführt. Ein transfemoraler Zugang erfolgt entweder via "cut down" oder perkutan. Ein Pigtail-Katheter (बAbb. 2c) wird im Aortenbogen für die Angiographie platziert $\left(45^{\circ}\right.$ - bis $75^{\circ}$-linksanteriooblique Projektion, [20]). Der Endograft ist an den beiden Enden nicht bedeckt, wobei dieser Teil über den Abgang der A. subclavia abgesetzt werden kann ( $\mathbf{0} \mathbf{A b b}$. 3b-d). Falls eine Überdeckung der linken A. subclavia (ca. $30 \%$ ) nötig ist, wird im Fall einer dominanten linken Vertebralarterie oder eines Koronararterien-Bypass mithilfe der A. mammaria interna vorgängig in derselben Sitzung eine Revaskularisation mit einem Karotis-Subklavia-Bypass durchgeführt (- Abb. 4c-g [20, 30]). 

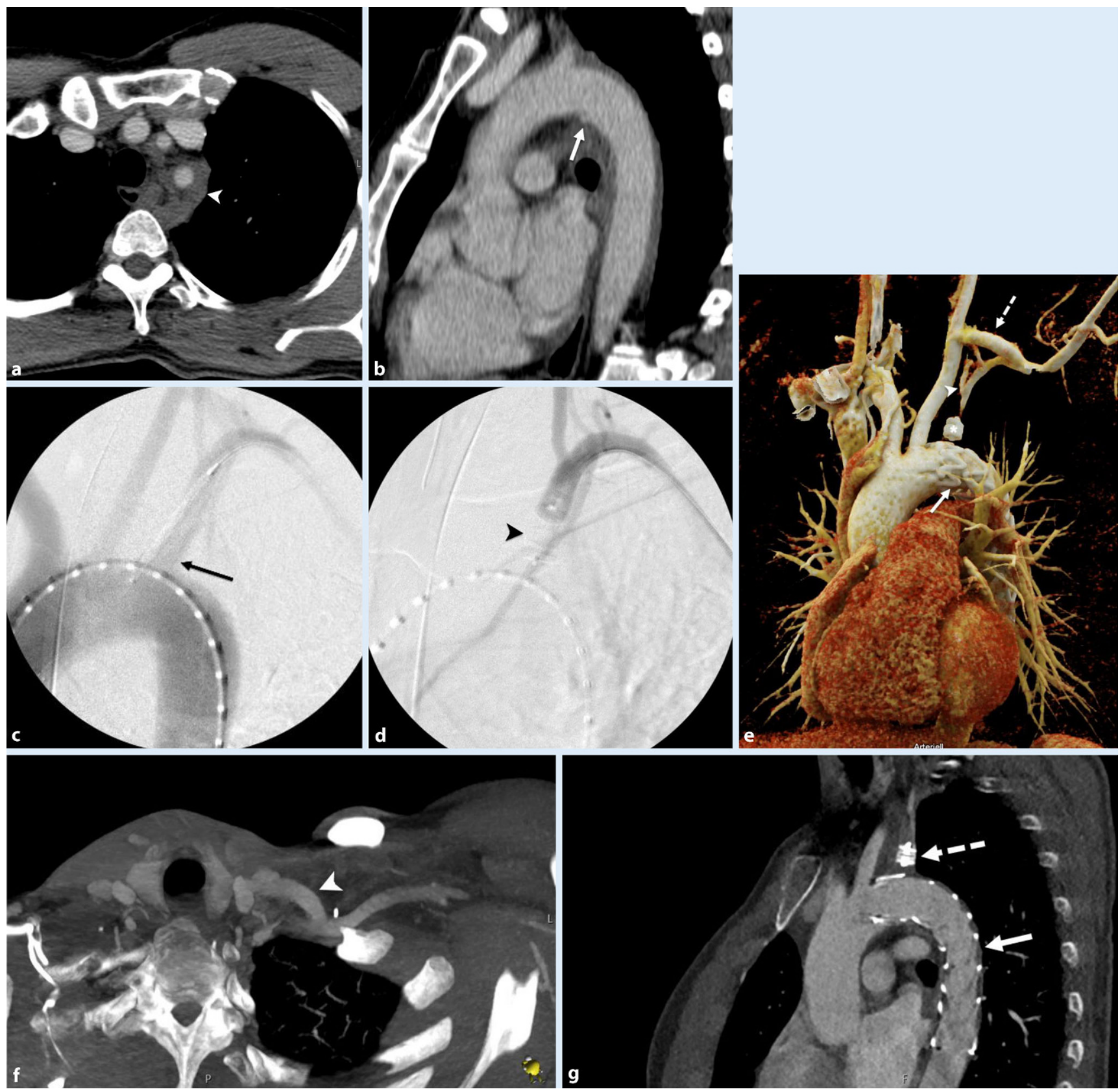

Abb. $4 \Delta$ Computertomographische Aufnahmen eines 39-jährigen Patienten nach Gleitschirmabsturz mit kleiner Wandaussackung loco typico (Pfeil) und Mediastinalhämatom (Pfeilspitze; a,b). Versorgung der Grad-IV-Verletzung mithilfe der "thoracic endovascular aortic repair" (TEVAR) und eines Karotis-Subklavia-Bypass. c,d Intraoperative fluoroskopische Aufnahmen vor (c) und nach (d) Okklusion der A. subclavia sinistra (Pfeil) am Ostium mithilfe eines „vascular plug“ (Pfeilspitze) zur Vermeidung eines Endoleak. e-f Unauffällige Befunde der Post-TEVAR-Kontrolle, in der 3D-Rekonstruktion (e) sind der StentKörper (Pfeil), der Vascular plug (Asteriskus) und der Karotis-Subklavia-Bypass (gestrichelter Pfeil) mit offener A. vertebralis sinistra (Pfeilspitze) über der retrograd perfundierten zentralen A. subclavia sinistra dargestellt. f Offener Karotis-SubklaviaBypass (Pfeilspitze) in den planaren Rekonstruktionen mit Vascular plug (gestrichelter Pfeil) in der A. subclavia sinistra und offenerTEVAR (g)

》) Die Größe des Endografts wird mit einem „oversizing" von 15-20\% gewählt
Zusätzlich wird der Abgang der A. subclavia embolisiert, falls das Risiko eines Endoleakgroß ist ([30];0 Abb.4d,e,g). Alternativ kann ein fenestrierter Graft in Erwägung gezogen werden, der in den letzten Jahren ebenfalls gute Resultate gezeigt hat [14,
27]. Der Endograft wird mit einem „oversizing" von $15-20 \%$ gewählt, das Oversizing kann aber im Notfall bis zu $30 \%$ betragen, falls keine passende Größe vorhanden ist [30]. Eine Heparinisierung sollte im Einzelfall und in der Zusammenschau mit den 
Begleitverletzungen erwogen werden, ist aber bei der Implantation eines KarotisSubklavia-Bypasses obligatorisch (mindestens $5000 \mathrm{IU}$ ). Der Endograft wird unter perpendikulärerer Fluoroskopie über einen supersteifen Draht an der proximalen Landezone positioniert. Nach angiographischer Kontrolle der Position wird dieser unter Apnoe freigesetzt [30, 34].

Falls in der Kontrollangiographie keine optimale Öffnung oder kein Kontakt zur Aortenwand ersichtlich ist, kann eine Angioplastie der proximalen und distalen Landezone durchgeführt werden [33].

Sollte die gesamte Aorta thoracica überstentet werden müssen, ist die Einlage einer spinalen Drainage zu erwägen, besonders wenn die distale Aorta betroffen ist. Dies verbessert die Durchblutung des Myelons durch Drucksenkung und verringert das Risiko für eine spinale Ischämie [33].

\section{Nachsorge}

Postintervention sollten die Patienten auf einer Intensivstation überwacht werden. Ein Monitoring der Vitalparameter und der neurologischen Funktion zum Ausschluss von zerebralen und spinalen Schlaganfällen sowie von Bein- und Mesenteriaischämien ist während 24 Stunden obligat [33]. Im Normalfall ist postinterventionell keine Heparinisierung notwendig.

Abhängig von Begleitverletzungen kann der Patient nach wenigen Tagen entlassen werden und normale physische Aktivität ist bereits nach wenigen Wochen wieder möglich [48].

Eine engmaschige Nachsorge mittels CTA (oder in speziellen Fällen mittels Magnet Resonanz Angiographie) nach 1, 6 und 12 Monaten und danach jährlich sollten im Verlauf durchgeführt werden, um ein Endoleak auszuschliessen [36, 48]. Im Falle eines Endoleaks sollte dieses entweder zeitnah angegangen werden (Typ I und III) oder engmaschiger kontrolliert werden (Typ II, IV und V) [4]. Ein positives Remodelling des Aortendurchmessers proportional zur Grösse des Oversizing des Stentgrafts ist dabei normal [2]. Bei jüngeren Patienten mit stabilem Bildbefund können die Kontrollintervalle im Verlauf verlängert werden, um eine exzessive Strahlenexposition zu vermeiden [22].
Resultate und Komplikationen Im Vergleich mit der chirurgischen Versorgung ist das perioperative Ergebnis der TEVAR besser; die Mortalität nach TEVAR beträgt 7,5-12\% und nach chirurgischen Verfahren 19-23\% [10, 11, 23, 32, 44]. Mögliche Komplikationen können durch den Stent-Graft oder durch die Intervention bedingt sein. Komplikationen durch den Stent-Graft treten in 4\% der Fälle auf und beinhalten Endoleaks, Graft-Versagen (Verschluss, Diskonnexion der Komponenten etc.), technisches Versagen oder eine Verletzung der Iliakalgefäße [3, 5, 29]. Ein Graft-Versagen kann Folge einer falsch gewählten Stent-Größe sein und auf einer Unterschätzung des Aortendurchmessers im CT wegen einer vorliegenden Hypotonie beruhen. Der Versuch, ein Oversizing zu verhindern, kann in der Wahl eines zu kleinen Stent-Graft-Durchmessers mit ebenfalls konsekutivem Graft-Versagen münden. Meist können diese Komplikationen aber mithilfe einer endovaskulären Versorgung behoben werden [34]. Die präoperative Planung, empfohlen auf der Basis einer EKG-getriggerten Dünnschicht-CTA, ist ein essenzieller Beitrag zum erfolgreichen Ergebnis [18, 45]. Letztlich spart eine gute bildbasierte TEVAR-Planung auch Interventionszeit ein.

Das Risiko einer Myelonischämie ist bei der TEVAR im Vergleich zur Chirurgie (3\% vs.9\%, [34]) deutlich reduziert und konnte mit Einführung von durchblutungsfördernden Maßnahmen [11] in einigen Studien bis auf $0 \%$ gesenkt werden $[10,11$, 32]. Das Risiko eines Schlaganfalls beträgt etwa $1-10 \%[8,44]$ und steigt bei Überdeckung der linken A. subclavia von 2,7 auf $4,7 \%$, wie eine Studie zur elektiven TEVAR zeigte [6].

\section{Resümee}

Die TAV ist eine seltene, aber umso häufiger letal verlaufende Verletzung, die unverzüglich diagnostiziert werden sollte, um die Mortalität zu senken. Die TEVAR als minimal-invasiver Eingriff wird in den meisten Guidelines, sofern nicht kontraindiziert, als Therapie der Wahl empfohlen.
Fazit für die Praxis

- Bei einer traumatischen Aortenverletzung (TAV) ist meist die thorakale Aorta betroffen.

- Eine frühe, schnelle und akkurate Diagnostik mithilfe der EKG-getriggerten CT und computertomographischen Angiographie (CTA) trägt entscheidend zur Reduktion der Mortalität bei.

- Für die Diagnose und Einteilung ist sowohl eine native als auch eine Kontrastmittel(KM)-gestützte Darstellung der Gefäße mithilfe der CTA elementar.

- Bei TAV der Grade II-IV ist eine invasive Therapie indiziert.

- Die Therapie der Wahl stellt die minimalinvasive thorakale endovaskuläre Aortenrekonstruktion (,thoracic endovascular aortic repair", TEVAR) unter Einbezug von anatomisch relevanten Punkten dar.

- Eine CTA-basierte 3D-TEVAR-Planung ist ein essenzieller Baustein in der Versorgung.

- Im Fall eines hämodynamisch stabilen Patienten sollte die TEVAR elektiv nach $24 \mathrm{~h}$ durchgeführt werden.

- Morbidität und Mortalität sind geringer als nach chirurgischen Verfahren.

\section{Korrespondenzadresse}

\section{Dr. R. M. Benz}

Diagnostische, Interventionelle und Pädiatrische Radiologie, Inselspital, Universität Bern

Freiburgstr. 18, 3010 Bern, Schweiz robyn.benz@insel.ch

Funding. Open access funding provided by University of Bern

\section{Einhaltung ethischer Richtlinien}

Interessenkonflikt. R.M. Benz, V. Makaloski, M. Brönnimann, N. Mertineit und H. von Tengg-Kobligk geben an, dass kein Interessenkonflikt besteht.

Für diesen Beitrag wurden von den Autoren keine Studien an Menschen oder Tieren durchgeführt. Für die aufgeführten Studien gelten die jeweils dort angegebenen ethischen Richtlinien.

Open Access. Dieser Artikel wird unter der Creative Commons Namensnennung 4.0 International Lizenz veröffentlicht, welche die Nutzung, Vervielfältigung, Bearbeitung, Verbreitung und Wiedergabe in jeglichem Medium und Format erlaubt, sofern Sie den/die ursprünglichen Autor(en) und die Quelle ordnungsgemäß nennen, einen Link zur Creative Commons Lizenz beifügen und angeben, ob Änderungen vorgenommen wurden.

Die in diesem Artikel enthaltenen Bilder und sonstiges Drittmaterial unterliegen ebenfalls der genannten 
Creative Commons Lizenz, sofern sich aus der Abbildungslegende nichts anderes ergibt. Sofern das betreffende Material nicht unter der genannten Creative Commons Lizenz steht und die betreffende Handlung nicht nach gesetzlichen Vorschriften erlaubt ist, ist für die oben aufgeführten Weiterverwendungen des $\mathrm{Ma}$ terials die Einwilligung des jeweiligen Rechteinhabers einzuholen.

Weitere Details zur Lizenz entnehmen Sie bitte der Lizenzinformation auf http://creativecommons.org/ licenses/by/4.0/deed.de.

\section{Literatur}

1. Alarhayem AQ, Rasmussen TE, Farivar B et al (2021) Timing of repair of blunt thoracic aortic injuries in the thoracic endovascular aortic repair era. J Vasc Surg 73:896-902

2. Alberta HB, Secor JL, Smits TC et al (2014) Comparison of thoracic aortic diameter changes after endograft placement in patients with traumatic and aneurysmal disease. J Vasc Surg 59:1241-1246

3. Bischoff MS, Geisbüsch P, Kotelis D et al (2013) Clinical significance of type II endoleaks after thoracic endovascular aortic repair. J Vasc Surg 58:643-650

4. Chen J, Stavropoulos SW (2015) Management of endoleaks. Semin intervent Radiol 32:259-264

5. Chen SW, Lee KB, Napolitano MA et al (2020) Complications and management of the thoracic endovascular aortic repair. Aorta (Stamford) 8:49-58

6. Cooper DG, Walsh SR, Sadat U et al (2009) Neurological complications after left subclavian artery coverage during thoracic endovascular aortic repair: a systematic review and metaanalysis. JVasc Surg 49:1594-1601

7. Creasy JD, Chiles C, Routh WD et al (1997) Overview of traumatic injury of the thoracic aorta. Radiographics 17:27-45

8. Dake MD, White RA, Diethrich EB et al (2011) Report on endograft management of traumatic thoracic aortic transections at 30 days and 1 year from a multidisciplinary subcommittee of the society for vascular surgery outcomes committee. JVasc Surg 53:1091-1096

9. Demehri S, Rybicki FJ, Desjardins B et al (2012) ACR appropriateness criteria $\left({ }^{\circledR}\right)$ blunt chest trauma-suspected aortic injury. Emerg Radiol 19:287-292

10. Demetriades D, Velmahos GC, Scalea TM et al (2008) Operative repair or endovascular stent graft in blunt traumatic thoracic aortic injuries: results of an American association for the surgery of trauma multicenter study. J Trauma 64:561-570 (discussion 570-561)

11. Demetriades D, Velmahos GC, Scalea TM et al (2008) Diagnosis and treatment of blunt thoracic aortic injuries: changing perspectives. J Trauma 64:1415-1418 (discussion 1418-1419)

12. Erbel R, Aboyans V, Boileau C et al (2014) 2014 ESC guidelines on the diagnosis and treatment of aortic diseases: document covering acute and chronic aortic diseases of the thoracic and abdominal aorta of the adult. The task force for the diagnosis and treatment of aortic diseases of the European society of cardiology (ESC). Eur Heart J 35:2873-2926

13. Fox N, SchwartzD, Salazar JHetal (2015) Evaluation and management of blunt traumatic aortic injury:

\section{Diagnostics and treatment of traumatic aortic injuries}

Background: Traumatic aortic injuries (TAI) are rare injuries in blunt thoracic trauma, which have a high morbidity and mortality. Rapid and accurate diagnosis as well as the correct choice of treatment are elementary for patient survival.

Objective: Determination of the current standards for diagnostics of TAl in the acute trauma setting and evaluation of the current guidelines for treatment.

Material and methods: A literature search was carried out for articles describing diagnostics of TAI. Furthermore, the guidelines for treatment and follow-up of TAI were summarized.

Results: Despite the low specificity conventional chest X-ray is still named in the literature as initial diagnostic procedure. Primarily, computed tomography (CT) should follow as the method of choice for diagnostics and treatment stratification due to the high sensitivity and specificity. Thoracic endovascular aortic repair (TEVAR) is recommended by all guidelines as first line treatment of higher grades of TAI (grades II-IV) and has replaced open surgery in most cases.

Conclusion: After rapid diagnosis and classification of TAI with CT, in most cases TEVAR has become the preferred treatment over open surgery.

\section{Keywords}

Classification · Angiography, computed tomography - Thoracic endovascular aortic repair - Open aortic surgery $\cdot$ Guidelines

a practice management guideline from the eastern association for the surgery of trauma. J Trauma Acute Care Surg 78:136-146

14. Gilani R, Ochoa L, Wall MJ Jr. et al (2011) Endovascular repair of traumatic aortic injury using a custom fenestrated endograft to preserve the left subclavian artery. Vasc Endovascular Surg 45:549-552

15. Hahn LD, Prabhakar AM, Zucker EJ (2019) Crosssectional imaging of thoracic traumatic aortic injury. Vasa 48:6-16

16. Hainer C, Böckler D, Bernhard M et al (2008) Blunt traumatic aortic injury: importance of transesophageal echocardiography. Anaesthesist 57:262-268

17. Hallinan JT, Anil G (2014) Multi-detector computed tomography in the diagnosis and management of acute aortic syndromes. World J Radiol 6:355-365

18. Hoegen P, Wörz S, Müller-Eschner M et al (2017) How precise are preinterventional measurements using centerline analysis applications? Objective ground truth evaluation reveals software-specific centerline characteristics. J Endovasc Ther 24:584-594

19. Igiebor OS, Waseem M (2021) Aortic trauma. StatPearls, Treasure Island (FL)

20. Kandarpa K, Machan L (2011) Handbook of interventional radiologic procedures. Lippincott Williams \& Wilkins,

21. Karmy-Jones R, Ferrigno L, Teso D et al (2011) Endovascular repair compared with operative repair of traumatic rupture of the thoracic aorta: a nonsystematic review and a plea for trauma-specific reporting guidelines. J Trauma 71:1059-1072

22. Katsargyris A, Verhoeven EL (2012) Part two: against the motion. All TEVAR patients do not require lifelong follow-up by annual CTA/MRA. Eur JVasc Endovasc Surg 44:538-541

23. Khoynezhad A, Azizzadeh A, Donayre CE et al (2013) Results of a multicenter, prospective trial of thoracic endovascular aortic repair for blunt thoracic aortic injury (RESCUE trial). J Vasc Surg 57:899-905.e1

$57.899-905 . e 1$
24. Khoynezhad A, Donayre CE, Azizzadeh A et al (2015) One-year results of thoracic endovascular aortic repairforblunt thoracicaorticinjury (RESCUE trial). JThorac Cardiovasc Surg 149:155-161.e4

25. Kotelis D, Bischoff MS, Jobst B et al (2012) Morphological riskfactors of stroke during thoracic endovascular aortic repair. Langenbecks Arch Surg 397:1267-1273

26. Kotelis D, Brenke C, Wörz S et al (2015) Aortic morphometry at endograft position as assessed by 3D image analysis affects risk of type I endoleak formation after TEVAR. Langenbecks Arch Surg 400:523-529

27. Kurimoto Y, Asai Y, Nara S et al (2009) Fenestrated stent-graft facilitates emergency endovascular therapy for blunt aortic injury. J Trauma 66:974-978 (discussion 978-979)

28. Lee WA, Matsumura JS, Mitchell RS et al (2011) Endovascular repair of traumatic thoracic aortic injury: clinical practice guidelines of the society for vascular surgery. JVasc Surg 53:187-192

29. Lioupis C, Mackenzie KS, Corriveau MM et al (2012) Midterm results following endovascular repair of blunt thoracic aortic injuries. Vasc Endovascular Surg 46:109-116

30. Makaloski V, Widenka H, Schönhoff F et al (2021) Efficacy and safety of heparinization before deployment of endograft for blunt traumaticaortic injury in severely injured patients. Ann Vasc Surg. https://doi.org/10.1016/j.avsg.2021.01.096

31. Mellnick VM, Mcdowell C, Lubner M et al (2012) CT features of blunt abdominal aortic injury. Emerg Radiol 19:301-307

32. Murad MH, Rizvi AZ, Malgor R et al (2011) Comparative effectiveness of the treatments for thoracic aortic transection. J Vasc Surg 53:193-199.e1-21

33. Nation DA, Wang GJ (2015) TEVAR: endovascular repair of the thoracic aorta. Semin Intervent Radiol 32:265-271

34. Neschis DG, Eidt JF, BulgerEM (2021) Management of blunt thoracic aortic injury 
35. Patel NR, Dick E, Batrick N et al (2018) Pearls and pitfallsinimaging ofblunt traumatic thoracicaortic injury: a pictorial review. Br J Radiol 91:20180130

36. Rengier $F$, Geisbüsch $P$, Schoenhagen $P$ et al (2014) State-of-the-art aortic imaging: part II-applications in transcatheter aortic valve replacement and endovascular aortic aneurysm repair. Vasa 43:6-26

37. Riambau V, Böckler D, Brunkwall J et al (2017) Editor's choice-management of descending thoracic aorta diseases: clinical practice guidelines of the European society for vascular surgery (ESVS). Eur JVasc Endovasc Surg 53:4-52

38. Schumacher H, Böckler D, von Tengg-Kobligk H et al (2006) Acute traumatic aortic tear: open versus stent-graft repair. Semin Vasc Surg 19:48-59

39. Sevitt S (1977) The mechanisms of traumatic rupture of the thoracic aorta. Br J Surg 64:166-173

40. Shalhub S, Starnes BW, Tran NT et al (2012) Blunt abdominal aortic injury. J Vasc Surg 55:1277-1285

41. Spanos K, Nana P, Behrendt CA et al (2021) Management of descending thoracic aortic diseases: similarities and differences among cardiovascular guidelines. J Endovasc Ther 28:323-331

42. Steenburg SD, Ravenel JG (2008) Acute traumatic thoracic aortic injuries: experience with 64-MDCT. AJR Am JRoentgenol 191:1564-1569

43. Steuer J, Björck M, Sonesson B et al (2015) Editor's choice-durability of endovascular repair in blunt traumatic thoracic aortic injury: long-term outcome from four tertiary referral centers. Eur J Vasc Endovasc Surg 50:460-465

44. Tang GL, Tehrani HY, Usman A et al (2008) Reduced mortality, paraplegia, and stroke with stent graft repair of blunt aortic transections: a modern metaanalysis. JVasc Surg 47:671-675

45. Teraa M, Hazenberg CE, Houben IB et al (2020) Important issues regarding planning and sizing for emergent TEVAR. J Cardiovasc Surg (Torino) 61:708-712

46. Theisen D, von Tengg-Kobligk $\mathrm{H}$, Michaely $\mathrm{H}$ et al (2007) CT angiography of the aorta. Radiologe 47:982-992

47. Upchurch GR Jr., Escobar GA, Azizzadeh A et al (2021) Society for vascular surgery clinical practice guidelines of thoracic endovascular aortic repair for descending thoracic aortic aneurysms. J Vasc Surg 73:55s-83s

48. Wang GJ, Fairman RM (2009) Endovascular repair of the thoracic aorta. Semin intervent Radiol 26:17-24

49. Wong S, Mastracci TM (2012) Part one: for the motion. All TEVAR patients must be followed lifelong by annual CTA/MRA. Eur J Vasc Endovasc Surg 44:534-537

\section{Knie Journal}

\section{Quadrizepssehnenruptur}

Eine seltene Verletzung!

i

Unfallmechanismus: Häufig durch ein indirektes Trauma mit

exzentrischer Belastung der Sehne.

Typischer Untersuchungsbefund: „suprapatellar gap sign“

Bildgebende Diagnostik

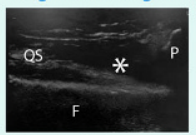
Diagnostik der Wah!!
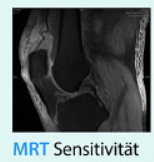
\& Spezifität $100 \%$

Ist Indikation zur Operation

OP möglichst innerhalb vo

2 Wochen nach Trauma

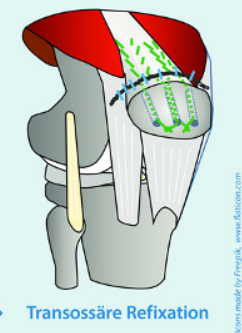

Springer Medizin
Die Quadrizepssehne ist die kräftigste Sehne des menschlichen

Körpers. Sie rupturiert meist ansatznah am oberen Patellapol auf

Basis degenerativer Veränderungen.

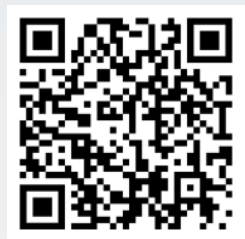

Lesen Sie weiter unter

www.SpringerMedizin.de/link/

10.1007/s43205-021-00108-w oder

nutzen Sie den QR-Code.

Diesen und weitere spannende Beiträge aus Ihrem Fachgebiet lesen Sie mit unserem Online-Abo e.Med. Dieses bietet Ihnen u.a. Volltextzugriff auf alle Zeitschriften Ihres Fachgebiets wie z.B. Der Unfallchirurg, Der Orthopäde, Obere Extremität, Operative Orthopädie \& Traumatologie, Knie Journal und viele weitere. Testen Sie das Angebot 14 Tage kostenfrei www.SpringerMedizin.de/eMed. 
Hier steht eine Anzeige.

黑 Springer 